\title{
Quasi-periodic variation of Peierls stress of dislocations in face-centered-cubic metals
}

\author{
Guisen Liu ${ }^{\mathrm{a}}$, Xi Cheng ${ }^{\mathrm{a}, \mathrm{b}}$, Jian Wang ${ }^{\mathrm{c}}$, Kaiguo Chen ${ }^{\mathrm{d}}$, Yao Shen ${ }^{\mathrm{a}}{ }^{*}$ \\ ${ }^{a}$ State Key Lab of Metal Matrix Composites, School of Materials Science and Engineering, \\ Shanghai Jiao Tong University, Shanghai 200240, P.R. China \\ ${ }^{\mathrm{b}}$ Department of Mechanical Engineering, Stanford University, CA94305, USA \\ ${ }^{\mathrm{c}}$ Mechanical and Materials Engineering, University of Nebraska-Lincoln, NE68588, USA \\ ${ }^{\mathrm{d}}$ Center for Compression Science, China Academy of Engineering Physics, Mianyang 621900, \\ P.R. China
}

\begin{abstract}
The Escaig stress, i.e. the shear stress perpendicular to the Burgers vector, modulates the stacking fault area between two partials of a full dislocation, in turn, affects the mobility of the dislocation. In this paper, using the newly improved semi-discrete variational Peierls-Nabarro (SVPN) model we studied the variation of Peierls stress $\left(\tau_{p}\right)$ of dislocations in face-centered-cubic crystals with respect to the Escaig stress. We found that $\tau_{p}$ quasi-periodically oscillates and the oscillation gradually decreases with the increase of Escaig stress. This quasi-periodic variation of $\tau_{p}$ can be mathematically described by the combination of a sinusoidal and an exponential function, and further accounted for by the variation of the stacking fault width (SFW) between two partials during their movement under applied stress. For the maximum $\tau_{p}$, SFW is about integral multiples of the Peierls period. For the minimum $\tau_{p}$, SFW is around half-integral multiples of Peierls period. The variation of $\tau_{p}$ is associated with the oscillation magnitude of SFW from half-integral multiples to integral multiples of the Peierls period and then back to integral multiples of Peierls period caused by the Escaig stress. Molecular dynamics (MD) simulations further examined quasi-periodic variation of $\tau_{p}$, validating the SVPN model's capability of predicting sophisticated behavior of dislocation under applied stress.
\end{abstract}

Keywords: Dislocation; Escaig stress; Peierls stress; Peierls-Nabarro model; Molecular dynamics.

* Corresponding author: Yao Shen, E-mail: yaoshen@sjtu.edu.cn 


\section{Introduction}

Dislocation motion is recognized as a crucial mechanism for facilitating plastic deformation in crystalline materials, while the mobility of dislocations is strongly influenced by their core structure and the applied stress (Cai et al., 2000; Srivastava et al., 2013; Stukowski et al., 2015). For instance, an increasing Escaig stress (the shear stress is on the slip plane and perpendicular to the Burgers vector) superimposes fluctuations to the otherwise linear increase of velocity for a screw dislocation in silicon when the glide force is proportionally increased at the same time (Cai et al., 2000). This is because the applied Escaig stress alternates the separation of the two partials between integral and half-integral multiples of period of the Peierls barrier. In addition to influencing dislocation mobility on the slip plane, the Escaig stress can also assist the cross slip of screw dislocations in face-centered-cubic (FCC) metals (Kang et al., 2014; Lu et al., 2004), by reducing the energy barrier through constricting the stacking fault on the primary slip plane (Kang et al., 2014; Lu et al., 2004) or expanding the stacking fault width on the cross slip plane (Kang et al., 2014).

Alternation of the dislocation core width, and thereby mobility and cross-slip of individual dislocations caused by the Escaig stress has great impact on the collective behaviors of dislocation assemble, and thereby on the plastic deformation mode and the resulting microstructures in FCC alloys, especially those with low stacking fault energy, such as austenitic stainless steels (Baudouin et al., 2013; Byun, 2003; Byun et al., 2006; Di Gioacchino and Quinta da Fonseca, 2015; Talonen and Hänninen, 2007). For example, when the separation between two partials dissociated from a full dislocation is moderate (less than 
$1 \mu \mathrm{m}$ ), the major plastic deformation mode is glide of perfect dislocations and the deformation microstructure is mainly associated with dislocation tangles plus stacking faults; while the separation is large (greater than $1 \mu \mathrm{m}$ ), the dominant deformation mechanism is glide of partial dislocations, and the resulted microstructure contains large numbers of stacking faults and twin bands (Byun, 2003) or micro-shear bands (shear band within a grain) (Talonen and Hänninen, 2007), depending on the testing temperature and the composition of the steel. In summary, the stress dependent dislocation properties and deformation-induced microstructures of FCC crystals rely much on the Escaig stress which changes core structure of dislocations, characterized by the separation of a pair of partial dislocations. However, little attention was paid to the correlation between the change of core structure and dislocation properties, i.e. how the Escaig stress affects the effective Peierls stress - the minimum stress to move the dislocation as a whole without thermal activation, especially for dislocations in FCC metals.

Atomistic simulation with empirical interatomic potentials is often adopted to study dislocation behavior under applied stress (Bulatov and Cai, 2006; Gröger and Vitek, 2013; Groh et al., 2009; Healy and Ackland, 2014; Rao et al., 2015; Salehinia and Bahr, 2014; Xiong et al., 2014; Xu et al., 2013; Yanilkin et al., 2014; Zhou et al., 2016), however, empirical potentials might be unavailable to some complex materials. Though first-principles density functional theory (DFT) can obtain the core structure of dislocations (Shin and Carter, 2014; Ventelon et al., 2013; Yadav et al., 2014), extended systems, such as dislocation motion under external loads, are too large to be handled in DFT calculations. Alternatively, the 
Peierls-Nabarro (PN) model (Nabarro, 1947; Peierls, 1940) becomes an attractive approach to study the core structure and the Peierls stress of dislocations, because it combines the efficiency of continuum description of elastic medium with the accuracy of generalized stacking fault surface which is calculated using atomistic models. Moreover, numerous improvements of the PN model (Bulatov and Kaxiras, 1997; Schoeck, 1994; Shen and Cheng, 2009; Vitek, 1968) have been proposed to overcome the shortcomings and inconsistency with the original model. These improved PN models have been examined and extended for predicting core structure and Peierls stress of dislocations in various crystals (Schoeck, 2012, 2013; Wang et al., 2014a), and even for Escaig stress assisted dislocation cross-slip in FCC metals (Lu et al., 2004). Particularly, the recently developed semi-discrete variational Peierls-Nabarro (SVPN) model (Liu et al., 2016) have been demonstrated to obtain a more accurate prediction of core structure and Peierls stress of dislocations in FCC metals, as well as the right trend in the variation of the stacking fault width as the dislocation overcomes the Peierls barrier. In addition, the improved SVPN model can also predict the dislocation profile much more efficiently than atomistic simulations by numerically minimizing the dislocation energy functional.

In this paper, we apply the improved SVPN model (Liu et al., 2016) to investigate the effects of the Escaig stress on core structure and Peierls stress of dislocations in FCC metals. We take the edge and screw dislocations in $\mathrm{Cu}$ as examples, because highly reliable interatomic potential is available for $\mathrm{Cu}$ (Mishin et al., 2001). We also perform MD simulations to exam the results obtained from the SVPN model (Liu et al., 2016). This paper 
is organized as follows. Section 2 describes theoretical background of Escaig stress effects, and computational details of both the improved SVPN model (Liu et al., 2016) and MD simulations. The results are presented and discussed in section 3, followed by a summary in section 4 .

\section{Models and simulation schemes}

The effect of Escaig stress on Peierls stress and core structure of a dislocation is studied by applying the improved SVPN model (Liu et al., 2016), aided by the nudged elastic band (NEB) methods (Henkelman and Jónsson, 2000; Henkelman et al., 2000) and MD simulations. The investigation begins by setting the dislocation geometry and theoretically analyzing how the Escaig stress affects dislocation core structure. Then, the improved SVPN model (Liu et al., 2016) is adopted to study the Escaig stress effects by directly incorporating it into the dislocation energy functional to calculate Peierls stress under different Escaig stress. Moreover, the NEB method is used to investigate the core structure change and partials interactions as the dislocation overcomes the Peierls barrier. Finally, MD simulations are performed to validate the predictions by the SVPN model.

\subsection{Dislocation geometry and stacking fault width}

Considering a full dislocation with Burgers vector $\vec{b}=a / 2[\overline{101}]$ on (111) glide plane in $\mathrm{Cu}, \quad$ the coordinate system in Fig. 1 is adopted, with $x$-, $y$ - and $z$ - (or 1, 2 and 3) axes along the crystal directions [1리, [111] and [101], respectively. The full dislocation dissociates into two Shockley partial dislocations connected by stacking fault, described as: $\vec{b}=\frac{a}{2}[\overline{1} 01] \rightarrow \frac{a}{6}[\overline{1} \overline{1} 2]+\frac{a}{6}[\overline{2} 11]+$ stacking faults, $a=3.615 \AA$ is the lattice constant. For the case of 
an edge dislocation, the dislocation line is along the $x$ axis, and the two partials are $60^{\circ}$ mixed type dislocations (Fig. 1a); and for the case of a screw dislocation, the dislocation line is along the $z$ axis, and the two partials are $30^{\circ}$ mixed type dislocations (Fig. 1b).
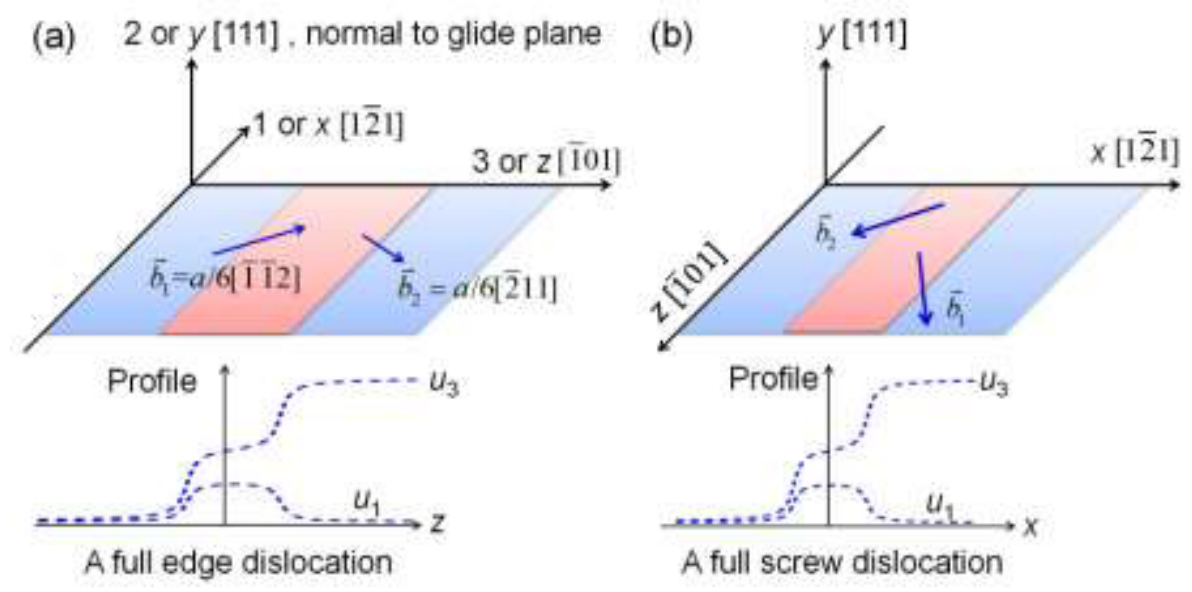

Fig. 1. Illustration of a full (a) edge and (b) screw dislocation dissociating into two Shockley partials on (111) plane connected with stacking faults. The full dislocation has Burgers vector $1 / 2$ [-101], parallel to $z$ axis. Dislocations' line is either parallel to $x$ (for edge dislocation) or $z$ (for screw dislocation) axis.

The core structure of the dislocation is characterized by slip profile denoted as $\mathbf{u}(z)$ for the edge dislocation and $\mathbf{u}(x)$ for the screw, which can be derived through disregistry analysis of the relative displacement of the atom pairs across the slip plane. Suppose the dislocation dissociation is constrained on the (111) plane (Fig. 1), then the profile vector $\mathbf{u}(z)$ or $\mathbf{u}(x)$ has two components $u_{1}$ and $u_{3}$, which are the disregistries along the $x$ - and $z$ - axis, respectively. Two components of the applied stress, $\tau_{y x}$ and $\tau_{y z}$, exert Peach-Koehler forces (Hirth and Lothe, 1982) on the two partials that are normal to the dislocation line:

$$
\begin{aligned}
& \text { Edge }: F_{1 z}=-\tau_{y z} b / 2-\tau_{y x} b_{1 x}, \quad F_{2 z}=-\tau_{y z} b / 2+\tau_{y x} b_{1 x} \\
& \text { Screw }: F_{1 x}=\tau_{y z} b / 2+\tau_{y x} b_{1 x}, \quad F_{2 x}=\tau_{y z} b / 2-\tau_{y x} b_{1 x}
\end{aligned}
$$


where $F_{i j}(i=1,2 ; j=x, z)$ are the Peach-Koehler force on partial dislocation $i$ along axis $j$, and $b_{1 x}=\sqrt{6} a / 12$ is the magnitude of $x$ component of the partial Burgers vector $\vec{b}_{1}$. It can be seen from Eq. (1) that the Escaig stress $\tau_{y x}$ exerts equal but of opposite signed forces on the two partials. Thus, the two partials can be pulled away or pushed close depending on the sign of $\tau_{y x}$. The Escaig stress results in zero Peach-Koehler force on the full dislocation. In contrast, the shear stress $\tau_{y z}$ (parallel to the full Burgers vector) exerts equal force on the two partials with the same sign, so moving the dislocation normal to its line direction.

When $\tau_{y z}=0$ ( see Fig. 1), the separation between the two partials can be readily calculated as a function of the Escaig stress $\tau_{y x}$ by the anisotropic linear elastic theory (Byun, 2003; Hirth and Lothe, 1982) :

$$
w\left(\tau_{y x}\right)=\left(H_{33}-\frac{1}{3} H_{11}\right) \frac{b^{2}}{2\left(\gamma_{i s f}-\tau_{y x} b_{1 x}\right)}
$$

where $H_{i j}$ is the component of the anisotropic Stroh tensor (Bacon et al., 1980), which is dependent on the orientation of the dislocation line and on the elastic constants; for $\mathrm{Cu} H_{11}=$ 6.05 GPa, $H_{33}=3.38 \mathrm{GPa}$ are employed for the screw dislocation and $H_{11}=3.55 \mathrm{GPa}, H_{33}=$ 5.94 GPa for the edge dislocation(Liu et al., 2016); $\gamma_{\text {isf }}$ is the intrinsic stacking fault energy (44 mJ/m² for $\mathrm{Cu}($ Liu et al., 2016)), which was obtained using embedded-atom method potentials (Mishin et al., 2001). According to Eq. (2), the separating distance monotonically increases with increasing Escaig stress. Furthermore, it also implies that there is a critical Escaig stress $\tau_{c}=\gamma_{i s f} / b_{1 x}$, above which the separation diverges to infinity [7], which will be discussed in section 3.1.

\section{2. Dislocation energy functions}


The total energy of the dislocation (Fig. 1) is a functional of the slip profile $\mathbf{u}(x)$ and its gradient $\nabla \mathbf{u}(x)$ (For edge dislocation, the displacement field $\mathbf{u}(x)$ would be replaced by $\mathbf{u}(z)$, and it is appropriate to use the same energy function form for both the screw and edge dislocations because their cores are spread on a slip plane) (Liu et al., 2016):

$$
U_{d i s}\left[\mathbf{u}(x), \tau_{\text {app }}\right]=\iint \frac{d \mathbf{u}(x)}{d x} \frac{\mathbf{H}}{x-x^{\prime}} \mathbf{u}\left(x^{\prime}\right) d x d x^{\prime}+\int \gamma[\mathbf{u}(x)] d x+\int \lambda\|\nabla \mathbf{u}(x)\|^{2} d x-\sum_{k} \int \tau_{k} u_{k}(x) d x
$$

where each term in the function is respectively elastic interaction energy between infinitesimal dislocations in the two half spaces, atomic misfit energy in the glide plane, gradient energy to account for the displacement gradient and discreteness effects, and work done by the external applied stress. $\boldsymbol{H}$ is the symmetric Stroh tensor (Bacon et al., 1980). $\gamma[\mathbf{u}(x)]$ is the generalized stacking fault (GSF) energy ( $\gamma$-surface). $\lambda$ is the gradient coefficient, dependent on the dislocation type (Liu et al., 2016), e.g. $0.25 \mu b(0.566 \mu b)$ for the screw (edge) dislocation in Cu. $\tau_{k}$ is the external applied stress component, with $\tau_{1}=\tau_{y x}, \tau_{3}=\tau_{y z}$ in the current coordinate system.

For the dislocation in Fig. 1, the discretized form of Eq. (3) is:

$$
\begin{aligned}
& U_{d i s}[\mathbf{u}(x)]=\sum_{k=1,3} \sum_{i=1}^{N} \sum_{j=1}^{N} H_{k} x_{i j} \rho_{k}\left(x_{i}\right) \rho_{k}\left(x_{j}\right)+\sum_{i=1}^{N} \gamma\left[u_{1}\left(x_{i}\right), u_{3}\left(x_{i}\right)\right] \Delta x \\
& +\lambda \sum_{k=1,3} \sum_{i=1}^{N}\left(\frac{u_{k}\left(x_{i+1}\right)-u_{k}\left(x_{i-1}\right)}{2 \Delta x}\right)^{2} \Delta x-\tau_{y z} \sum_{i=1}^{N}\left[u_{3}\left(x_{i}\right)+u_{3}\left(x_{i+1}\right)\right] \Delta x / 2-\tau_{y x} \sum_{i=1}^{N}\left[u_{1}\left(x_{i}\right)+u_{1}\left(x_{i+1}\right)\right] \Delta x / 2
\end{aligned}
$$

where $\Delta x=x_{i+1}-x_{i}$ is the discrete node spacing, being either $\sqrt{3} b / 2$ (screw) or $b$ (edge); $N$ is the number of the total nodes used in this calculation, being 200; $\rho_{k}\left(x_{i}\right)=\left(u_{k}\left(x_{i+1}\right)-u_{k}\left(x_{i+1}\right)\right) / \Delta x$ is the component of the dislocation density ( $k=1$ is the $x$ component, and 3 the $z$ component); $\chi_{i j}=\frac{3}{2} \phi_{i+1, i} \phi_{j+1, j}+\psi_{i, j}+\psi_{i+1, j+1}-\psi_{i+1, j}-\psi_{i, j+1}$, with $\phi_{i, j}=\left(x_{i}-x_{j}\right), \quad \psi_{i, j}=\frac{1}{2}\left(x_{i}-x_{j}\right)^{2} \ln \left|x_{i}-x_{j}\right| ; H_{k}$ is the component of Stroh tensor $\left(H_{1}=H_{11}, H_{3}=H_{33}, H_{13}=0\right.$ for $\mathrm{Cu}$ (Liu et al., 2016)); the last 
term in Eq. (4) describes the interaction of the Escaig stress with the dislocation; $\gamma\left[u_{1}(x), u_{3}(x)\right]$ is the GSF energy, which is obtained by quadratic serendipity shape function (Taylor, 1972) interpolating between $\gamma$-surface calculated from molecular statics simulations. Details of calculating $\gamma$-surface of the (111) plane for Cu by molecular statics simulations are referred to the supplementary materials in (Liu et al., 2016).

\subsection{Solution scheme for Peierls stress $\tau_{p}$}

Starting from the energy function in Eq. (4), Peierls stress $\tau_{p}$ under various Escaig stress was calculated in four steps: (1) determining the gradient coefficient $\lambda$; (2) calculating the core structure (slip profile) at zero applied stress $\left(\tau_{y x}=\tau_{y z}=0\right.$ ); (3) calculating the core structure (slip profile) at increasing Escaig stress and zero glide stress ( $\left.\tau_{y x} \neq 0, \tau_{y z}=0\right)$; and (4) determining $\tau_{p}$ at the presence of various Escaig stress.

The gradient coefficient $\lambda$ is determined by calibrating the dislocation's equilibrium profile obtained by the modified SVPN model against the one by MD simulation, and the value of $\lambda$ is obtained by minimizing the sum of the squared errors of the two equilibrium slip profiles at zero stress (Liu et al., 2016). In addition, the slip profiles at any $\lambda$ computed by the improved SVPN model is ensured to be in the global energy minimum site by combining the steepest descent method and the Nudge Elastic Band (NEB) methods, same way as obtaining the equilibrium slip profiles at zero applied stress in the next.

To obtain the slip profile at zero applied stress, we firstly solved the profile by minimizing the total energy of Eq. (4) with a simple steepest descent method, when the step function (a Volterra dislocation) was taken as the initial trial profile, and the two ends of the 
slip profile were clamped, and the profiles in between the fixed ends were allowed to vary in the energy minimization (Liu et al., 2016). The solved profile is in turn taken as the initial guess in the second round search for the lowest energy dislocation core structure. The second round search is to find the minimum energy path (MEP) of translating the profile by a Peierls period using NEB methods, with the slip profile determined in the first search as the ending points of the MEP, and then take the configuration (slip profile) at the valley of the MEP as the equilibrium profile under zero applied stress. The rationale of employing such a two-round search is that the equilibrium dislocation profile should stay in the Peierls valley, but a dislocation can be trapped in a local energy minimum by the simple steepest descent search if the initial guess is far away from the global minimum. It is noteworthy that the slip profile at the global energy minimum is slightly wider (less than one Peierls period) than the one obtained by MD calculations. This is the reason for the difference of stacking fault width obtained from the SVPN model and MD simulation in section 3.2 and 3.3.

The Escaig stress is then applied to the above determined equilibrium profile at the global minimum energy site, e. g. increasing $\tau_{y x}$ from zero to -300 or $300 \mathrm{MPa}(0.00728 \mu)$ in Eq. (4), and the equilibrium profile (core structure) under each Escaig stress is obtained by minimizing the total energy of Eq. (4) by taking the slip profile at a smaller applied stress as the initial guess using the steepest search.

To determine $\tau_{p}$, glide stress is incrementally applied to the solved equilibrium profiles from the step described above under different Escaig stress, e.g. increasing $\tau_{y z}$ from zero to a critical value in Eq. (4), and then minimizing the energy again to obtain the equilibrium 
profile at increasing glide stress for each Escaig stress. Fig. 2a shows the variation of the dislocation profiles as applied glide stress $\tau_{\mathrm{yz}}$ increases from zero to above a critical value (13 $\mathrm{MPa})$. Below the critical glide stress, the dislocation profile slightly changes its shape without evident displacement (much less than one lattice period), but once the applied glide stress exceeds the critical value, a significant translation of the dislocation profile occurs (on the order of several lattice periods) (Cheng et al., 2012; Liu et al., 2016; Shen and Cheng, 2009). This critical glide stress is taken as the Peierls stress.
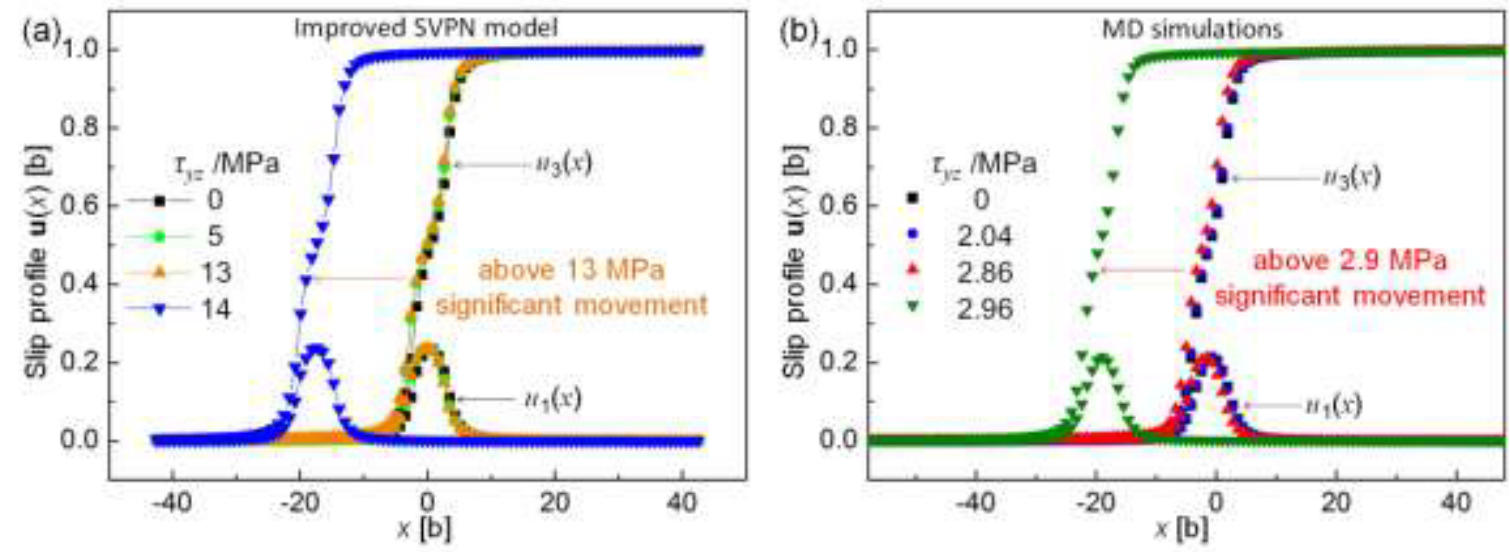

Fig. 2. Variation of the slip profiles of the screw dislocation in $\mathrm{Cu}$ at zero Escaig stress with increasing glide stress $\tau_{y z}$. Peierls stress was determined to be (a) $13 \mathrm{MPa}$ by the improved SVPN model, and (b) 2.9 MPa by MD simulations, respectively.

\subsection{Nudged elastic band methods for the minimum energy path}

The NEB methods (Henkelman and Jónsson, 2000; Henkelman et al., 2000) were used in both SVPN model and MD simulations to find the MEP for the full dislocation to move from on Peierls valley to an adjacent one, and to investigate the interactions between the two partials along the MEP. The performance of MD simulations is to validate the SVPN model, 
and this can be accomplished in three steps.

The first step is to calculate two stable states of the dislocation located at two neighboring Peierls valleys (separated by one Peierls period $\Delta x$ ), which are the fixed two ends of the MEP (the initial and final states in the NEB calculations). In the SVPN model, the profiles of the two stable states are both fixed, while in MD, atoms in the initial state are fixed, but atoms in the final state are allowed to slightly adjust their position near the stable states to minimize the system energy.

The second step is to linearly interpolate 31 images between the initial and final states in SVPN model (MD) and then minimize the total force for each image. When the maximum total force of all interpolated images is below a tolerance force (per unit length), e.g. $10^{-5} \mathrm{~N} / \mathrm{m}$, the chain of states is considered as a representation of the MEP for the dislocation moving from one Peierls valley to the next.

The final step is to analyze the slip profiles of all the images obtained along the MEP to acquire the dislocation core information, such as variations of dislocation center and SFW along the MEP. So the interaction between the partials can be further analyzed, as will be displayed in section 3.2.

Additionally, the max slope of the MEP, i.e. $\frac{1}{b}\left(\frac{d E}{d x_{c}}\right)_{\max }$ is an alternative way to define Peierls stress, where $E$ is the energy variation as the dislocation moves, and $x_{c}$ is the displacement of the dislocation center, which is determined as the position of $u_{3}\left(x_{c}\right)=0.5 b$.

2.5. Molecular dynamics simulations for the effects of Escaig stress on the Peierls stress

To validate the SVPN predictions for the Escaig stress effects on core structure and the 
Peierls stress, we performed MD simulations for screw dislocations as follows.

(1) A simulation box has the dimensions, $106.26 \mathrm{~nm}$ in the $x$-axis ([1-21]), $112.70 \mathrm{~nm}$ in the $y$-axis ([111]), and $1.53 \mathrm{~nm}$ in the $z$-axis ([-101]). Periodic boundary condition was applied along the dislocation line direction ( $\mathrm{z}$ axis), while the other two directions were fixed boundary conditions by immobilizing several layers of atoms. The dislocation was introduced in the center of the simulation box according to its isotropic displacement field following linear elastic theory (Hirth and Lothe, 1982), followed by minimizing the potential energy to reach the equilibrium state. More details can be found in the supplementary materials in (Liu et al., 2016). Equilibrium slip profile (see Fig. 2b for the profile under zero applied stress) was obtained through disregistry analysis.

(2) The Escaig stress was applied to the simulation box by shear deformation at a constant strain rate of $\dot{\gamma}_{y x}=10^{8} / \mathrm{s}$, followed by relaxing the system in canonical ensemble at temperature of $2.5 \mathrm{~K}$ for 10 picoseconds, and then conjugate gradient method was used to minimize the total energy at zero temperature.

(3) Peierls stress is determined in the same manner as in the improved SVPN model, namely determined as a critical glide stress above which a significant translation of dislocation profile occurs. Glide stress is applied to the fully relaxed system under a certain Escaig stress at a constant strain rate of $\dot{\gamma}_{y z}=10^{7} / \mathrm{s}$, and the total energy is minimized after each increment $\left(\Delta \tau_{y z}=0.1 \mathrm{MPa}\right)$. Fig. 2b shows the equilibrium slip profiles at zero Escaig stress with increasing glide stress, from which Peierls stress is determined as $2.9 \mathrm{MPa}$.

\section{Results and discussion}


This section firstly summarizes the variations of Peierls stress with respect to increasing Escaig stress, followed by the relationship between Peierls stress and core structure (SFW). Then we analyze the change of dislocation core structures and interactions between the two partials as the dislocation overcomes the Peierls barrier under the application of Escaig stress, so as to understand the underlying mechanism of the effects on Peierls stress by Escaig stress. Validity of results predicted by the SVPN model is discussed in the end.

\subsection{Quasi -periodic variations of the Peierls stress w. r. t. the Escaig stress}

Variation of the Peierls stress $\tau_{p}$ with respect to the Escaig stress $\tau_{y x}$ is studied by applying the improved SVPN model (Liu et al., 2016) to determine $\tau_{p}$ in a range of $\tau_{y x}$ from -300 MPa to $300 \mathrm{MPa}(0.00728 \mu)$ for a screw dislocation, or to $200 \mathrm{MPa}(0.00485 \mu)$ for an edge dislocation. The results are summarized in Fig. 3, which shows that $\tau_{p}$ quasi-periodically varies from a local maximum value to a neighboring local minimum. Furthermore, the local maximum values decrease and the local minimum increase with increasing $\tau_{y x}$. MD simulations produce a similar relationship between $\tau_{p}$ and $\tau_{y x}$ (see the red curve in Fig. 3a), showing a gradually decreasing peak values and increasing valley values for $\tau_{p}$. It is also noted that the two curves display the same phase in variation, i.e. the values of $\tau_{y x}$ at which $\tau_{p}$ reaches local maximum/minimum are roughly the same for the two models, although the magnitude of $\tau_{p}$ obtained by the SVPN model is about five times those by MD simulations. This several times higher prediction of Peierls stress by the improved SVPN model, is partially due to the simplifications made in the Peierls-Nabarro model, i.e. the interatomic interaction is confined in the slip plane. However, the quasi-periodic variation of Peierls 
stress under increasing Escaig stress was not affected by this several times difference. In addition, $\tau_{p}$ of the edge dislocation at zero Escaig stress calculated by the improved SVPN (4 MPa, $9.7 \times 10^{-5} \mu$ ), is in much closer agreement with the experiment estimated about $0.5 \mathrm{MPa}$ $\left(1.2 \times 10^{-5} \mu\right)$ (Kamimura et al., 2013), compared with 69 MPa predicted by the previous SVPN model (Liu et al., 2016).
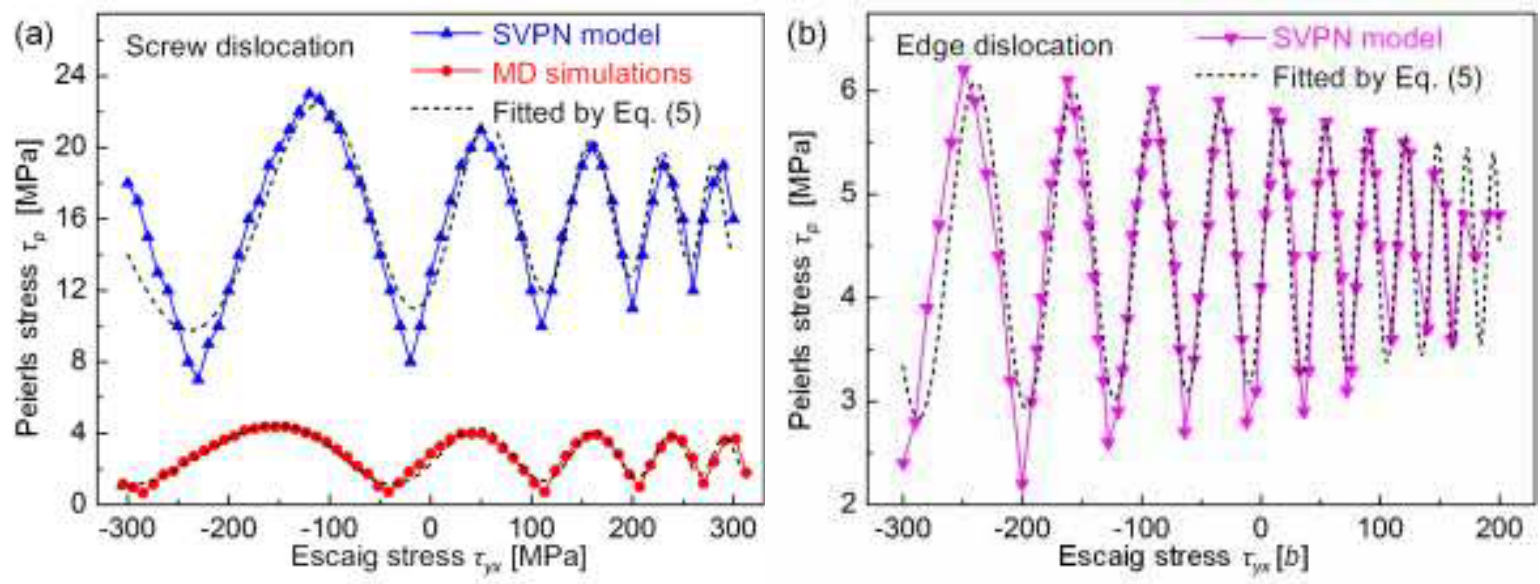

Fig. 3. Variation of Peierls stress with increasing Escaig stress. (a) A screw and (b) an edge dislocation in $\mathrm{Cu}$. Predictions by the improved SVPN model and MD are fitted by Eq. (5) and shown in dashed curves.

Bearing in mind the two features of the variation of $\tau_{p}$ with respect to Escaig stress $\tau_{E S}$ $\left(\tau_{y x}\right)$ revealed by the SVPN and MD simulations in Fig. 3, and the general exponentially decaying trend of $\tau_{p}$ with dislocation width (Peierls, 1940; Shen and Cheng, 2009), we find an analytical expression combining an exponential function and a sinusoidal function can well describe the Escaig stress effects on $\tau_{p}$ :

$$
\tau_{p}\left(\tau_{E S}\right)=\tau_{1} \exp \left[-\alpha /\left(1-\tau_{E S} / \tau_{c}\right)\right] \sin \left[\beta /\left(1-\tau_{E S} / \tau_{c}\right)\right]+\tau_{0}
$$

where $\tau_{0}, \tau_{1}, \alpha, \beta$ are fitting parameters, while $\tau_{c}$ is physically based on properties of the 
crystals: it is the critical Escaig stress to completely separate the two partials (Byun, 2003), and equal to $596.3 \mathrm{MPa}(0.01447 \mu)$ for the dislocations (see Fig. 1) in copper. All the parameters for the fitting curves are listed in Table 1, for edge and screw dislocations in copper.

Table 1. Coefficients for the Escaig stress effects on Peierls stress in Eq. (5).

\begin{tabular}{ccccc}
\hline & $\tau_{0}[\mathrm{MPa}]$ & $\tau_{1}[\mathrm{MPa}]$ & $\alpha$ & $B$ \\
\hline edge, SVPN & 4.486 & 2.696 & 0.7278 & 81.42 \\
screw, SVPN & 16.42 & 11.55 & 0.7689 & 24.15 \\
screw, MD & 2.768 & -2.450 & 0.4451 & 21.68 \\
\hline
\end{tabular}

The sinusoidal term in Eq. (5) describes the periodic variation of $\tau_{p}$, and the exponential term reflects the decreasing amplitude of the oscillation as Escaig stress increases. The term $\left(1-\tau_{E S} / \tau_{c}\right)^{-1}$ inside the exponential and sinusoidal terms reflects the variation of stacking fault width (roughly equal to dislocation width for dissociated dislocations) with respect to the Escaig stress, as can be seen in Eq. (6), which is actually a transform of Eq. (2):

$$
w\left(\tau_{y x}\right)=\frac{\left(H_{33}-H_{11} / 3\right) b^{2}}{2 \gamma_{i s f}\left(1-\frac{\tau_{y x}}{\gamma_{i s f} / b_{1 x}}\right)}=\frac{\left(H_{33}-H_{11} / 3\right) b^{2}}{2 \gamma_{i s f}\left(1-\tau_{y x} / \tau_{c}\right)} \propto\left(1-\tau_{y x} / \tau_{c}\right)^{-1}
$$

This indicates that the oscillation amplitude exponentially depends on dislocation width/stacking fault width, similar to the Peierls stress dependence on dislocation width (Peierls, 1940; Shen and Cheng, 2009), and the period of the oscillation inversely scales with the dislocation width/stacking fault width.

The parameter $\tau_{0}$ in Eq. (5) could be interpreted as the Peierls stress for a single partial dislocation. The rationale is that Eq. (5) implies that $\tau_{p}$ converges to $\tau_{0}$ when the Escaig stress reaches or exceeds the critical stress $\tau_{c}$ so that the interaction between the two partials 
disappears due to the infinite separation. Consequently, the Peierls stress under such condition can be viewed as that of a partial dislocation, as well as a full dislocation. From this point of view, the terms other than $\tau_{0}$ in Eq. (5) reflect the effects of the interaction between the two partials on the Peierls stress. When $\tau_{E S}=0$, the interaction part weighs $8 \%$ of the total $\tau_{p}$ for edge dislocation and $37 \%$ for screw dislocation. The lower weight implies weaker interaction in edge dislocation, in accordance with wider dislocation width of an edge dislocation than that of a screw dislocation.

In addition, the results obtained by the improved SVPN model for a screw dislocation in $\mathrm{Cu}$ (see Fig. 3a) indicate that the ratio of local minimum $\tau_{p}$ over its right neighboring local maximum increases from about $30 \%$ to $65 \%$. This predicted ratio agrees closely with the results by atomistic simulations of the effective Peierls stress for a screw dislocation in nickel (Szelestey et al., 2005), which shows that a local minimum $\tau_{p}$ under certain Escaig stress is about one third of a local maximum $\tau_{p}$.

More than calculating $\tau_{p}$ and core structure under only a few typical Escaig stress by atomistic simulations (Szelestey et al., 2005), a fitting of the effects of $\tau_{E S}$ on $\tau_{p}$ is given by Eq. (5). This quantitative relationship is obtained by calculating $\tau_{p}$ in a broad range of $\tau_{E S}$, and comprehensively analyzing the effects of Escaig stress on $\tau_{p}$ and core structure. Such variations of $\tau_{p}$ imply that, when $\tau_{p}$ is inputted as lattice friction in continuum modeling, such as dislocation dynamics simulations (Cui et al., 2016; Lyu et al., 2015), and crystal plasticity model (Amodeo et al., 2016; Lin et al., 2016; Lyu et al., 2015), the effects of external stress on $\tau_{p}$ should also be appropriately accounted for (Amodeo et al., 2016). 


\subsection{The Equilibrium stacking fault width (SFW) at the Peierls stress extremes}

To understand the Escaig stress effects on $\tau_{p}$, the equilibrium stacking fault width (SFW) is computed at Escaig stress correspond to $\tau_{p}$ extremes (either local maximum or minimum $\tau_{p}$ ). SFW, the key quantity to characterize core structure, is determined as the distance between the two peaks of the dislocation density (see Fig. 4) in the Burgers vector direction $\left(\rho_{3}=\right.$ $d u_{3} / d x$ ), which is calculated from the discrete slip profiles with cubic interpolation. Table 2 lists the SFWs determined from the stable slip profiles at zero Escaig stress through the improved SVPN or MD simulations, which are close to experimental results (Stobbs and Sworn, 1971) for both screw and edge dislocations.

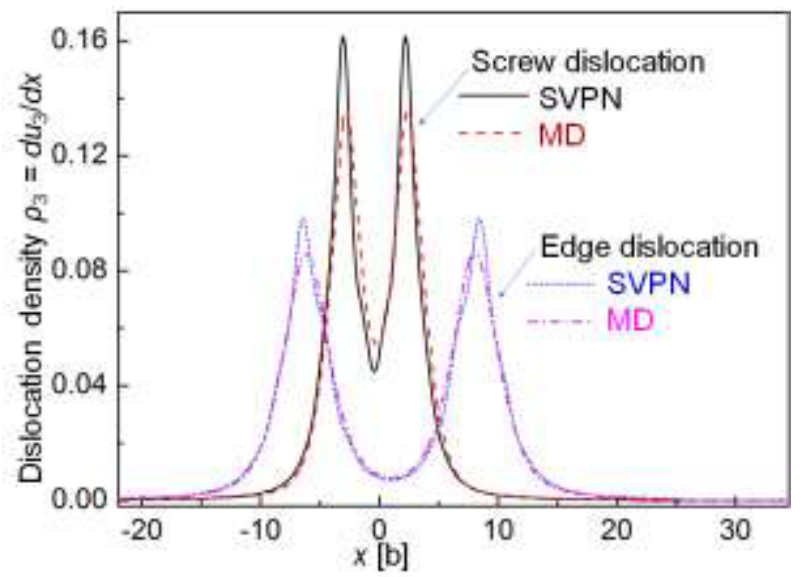

Fig. 4. Dislocation density for the screw and edge dislocation at zero applied stress predicted by improved SVPN model and MD simulations.

Table 2. Separation between the two partial dislocations at zero applied stress.

\begin{tabular}{cccc}
\hline & \multicolumn{3}{c}{$w$ or SFW (in units of b) } \\
\cline { 2 - 4 } & MD & Improved SVPN & Experiment value (Stobbs and Sworn, 1971) \\
\hline Cu, screw & 5.2 & 5.9 & $\sim 7.0$ \\
Cu, edge & 14.4 & 14.8 & $\sim 14.9$ \\
\hline
\end{tabular}

We find SFWs at the local maximums of $\tau_{p}$ are centered around integral multiples of 
Peierls period $\Delta x$, while at the local minimums of $\tau_{p}$, most of SFW are centered around half-integral multiples of $\Delta x$. This is illustrated in Fig. 5 for both screw and edge dislocations, where $\varphi_{n}\left(\varphi_{n+0.5}\right)$ is defined as the deviation of SFW to its nearest integral (half-integral) multiples of $\Delta x$. Most of the deviations are less than $\pm 0.25 \Delta x$, except that SFWs at some local minimums of $\tau_{p}$ of the screw dislocation calculated by the SVPN is slightly closer to integral multiples of $\Delta x$ than to half-integral multiples of $\Delta x$.
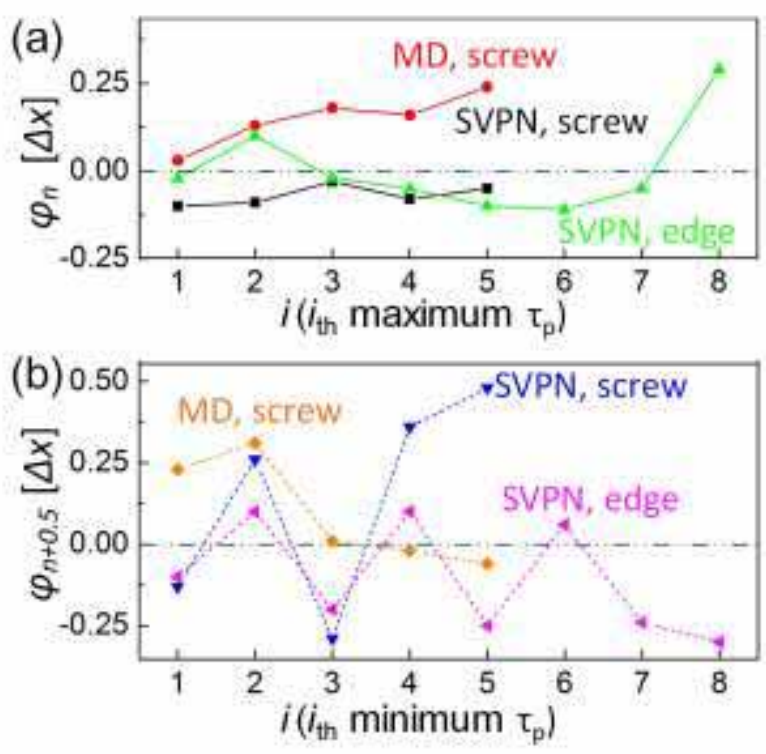

Fig. 5. Deviation of SFW to its nearest (a) integral multiples of Peierls period for the $i_{t h}$ local maximum Peierls stress and (b) half-integral multiples of Peierls period for $i_{t h}$ local minimum Peierls stress.

\subsection{Interactions of two partials}

Further, the interaction between the two partials as a full screw dislocation moves over the Peierls barrier is analyzed by examining the variation of SFW during this course. Two representative cases are studied in detail: (1) $\tau_{p}$ reaches a local maximum ( $\tau_{p}=21 \mathrm{MPa}, \tau_{E S}=$ 
$50 \mathrm{MPa}$ and equilibrium SFW $=7 \Delta x)$, and (2) $\tau_{p}$ reaches the neighboring local minimum $\left(\tau_{p}=\right.$ $10 \mathrm{MPa}, \tau_{E S}=110 \mathrm{MPa}$, and equilibrium SFW = 7.21 $\Delta x$ ). These two typical cases have also been investigated with MD simulations to further validate the predictions by the SVPN model.

Distinct differences exist for the interaction between the two partials, as evidenced by the different variations of SFW along MEP for the dislocation to advance one Peierls period (Fig. 6). In the case where $\tau_{p}$ reaches local maximum at $\tau_{E S}=50 \mathrm{MPa}$, the SFW changes little and remains nearly constant at $7 \Delta x$ as the dislocation moves (see Fig. 6a). It means both partials rest at Peierls valley at zero glide stress and start to move in phase as the glide stress increases. While in the case where $\tau_{p}$ reaches the neighboring local minimum at $\tau_{E S}=110$ MPa, the SFW shows large variation up to about $0.5 \Delta x$ : expanding from $7.2 \Delta x$ to $7.7 \Delta x$ and then shrinking back to $7.2 \Delta x$ (see Fig. 6a). This implies that when the SFW changes to around $7.5 \Delta x$, the two partials are in opposite phase: one partial is climbing up the Peierls barrier while the other is descending, and the leading partial moves first and the trailing partial catches up in sequence. This pattern of SFW variation has been reported for screw dislocation in nickel (Szelestey et al., 2005), where the two partials can move either in phase (SFW is near integral multiples of Peierls period) or in anti-phase (SFW is close to half-integral multiples of Peierls period), which accounted for about $70 \%$ reduction in the effective Peierls stress. 

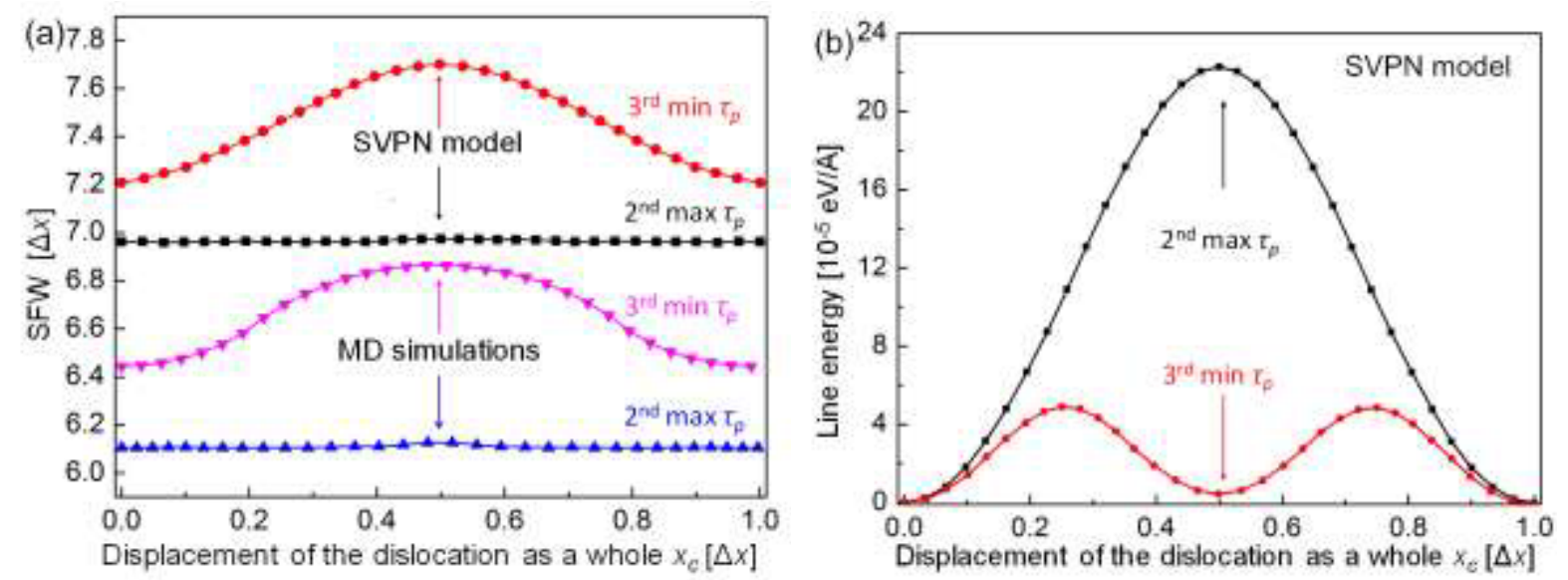

Fig. 6. Variations of (a) SFW and (b) dislocation energy for the screw dislocation moving one Peierls period $(\Delta x=\sqrt{3} b / 2)$ in copper for two representative cases when Peierls stress reaches local maximum and minimum respectively. Note that we plot SFW in (a) from 5.9 $\Delta x$ to $7.9 \Delta x$ to better illustrate the relatively small magnitude of variation of SFW.

The distinct characteristics of partial dislocations interactions and the predicted SFW variations can explain the existence of local maximum/minimum Peierls stress (see Fig. 3). When the two partials move in phase, both partials must overcome their individual and commensurate Peierls barrier simultaneously (Schoeck and Püschl, 1994), therefore a maximum Peierls barrier occurs (Fig. 6b), and consequently a local maximum $\tau_{p}$. But if the two partials become in opposite phase at nearly half way in the transition process, the movement of one partial climbing up the Peierls hill is aided by the other descending down the the Peierls hill (Schoeck and Püschl, 1994). In such case, cooperation between the partials can reduce the Peierls stress to larger extent and a local minimum $\tau_{p}$ occurs. These characteristics of energy variation along the MEP as shown in Fig. $6 \mathrm{~b}$ for the two extreme $\tau_{p}$ are similar to that of the effective Peierls potential reported by Schoeck (Schoeck and Püschl, 
1994), which was featured by a single high hump vs. two low humps. The good agreement between the SVPN model and atomistic simulations about the predictions for the Peierls stress of screw dislocation under typical SFWs (either integral or half-integral multiples of $\Delta x$ ) indicates that the SVPN model can well capture the complex interaction between the two partials under Escaig stress from another perspective.

Inspection of the variation of SFW along MEP in all the other cases of $\tau_{p}$ extremes (either local maximum or minimum $\tau_{p}$ ) confirms the observation that local maximum $\tau_{p}$ occurs when the two partials are nearly in phase when they climb the steepest portion of the Peierls barrier, and local minimum $\tau_{p}$ occurs when the two partials are in opposite phase at nearly half way of the transition process. In the former case the SFW is approximately integral multiples of Peierls period while the latter it is about half-integral multiples of Peierls period when zero glide stress is applied. When SFW changes through a sequence of integral and half-integral multiples of Peierls period caused by the increasing Escaig stress, as implied in Eq. (2), it is expected that $\tau_{p}$ periodically varies from a local maximum to a minimum. Furthermore, interactions between the two partials become weaker as SFW increases, hence reducing difference between the maximum and minimum $\tau_{p}$, and the two extremes should converge to $\tau_{0}$, as discussed in section 3.1. Therefore, the predicted Quasi-periodic variation of the Peierls stress with the Escaig stress (see Eq. (5)) can be explained by the above analysis of the two partials interaction.

\section{4 Validity of the prediction by the SVPN model}

The validation of the improved SVPN model has been presented in an earlier study (Liu 
et al., 2016) for zero Escaig stress condition, and is further discussed in this section for more complex condition where Escaig stress is applied. First, accuracy of the Peierls stress under Escaig stress predicted by the SVPN model is evidenced by two facts: (1) The quasi-periodic variation of Peierls stress of the screw dislocation in $\mathrm{Cu}$ (see Fig. 3a) predicted by SVPN is similar to that of MD simulations; (2) $\tau_{p}$ determined as the critical glide stress to move the dislocation forward (see Fig. 2a) in the SVPN model is consistent with that determined from the maximum slope of the MEP as can be seen in Table 3 for the two typical Escaig stress cases as discussed in section 3.3.

Table 3. Peierls stress for the screw dislocation in $\mathrm{Cu}$ under two typical Escaig stress $\tau_{y x} \cdot$

\begin{tabular}{cccc}
\hline \multirow{2}{*}{$\tau_{y x}($ in MPa $)$} & \multicolumn{2}{c}{$\tau_{p}$ (in MPa) } \\
\cline { 3 - 4 } & & critical glide stress & NEB method \\
\hline \multirow{2}{*}{ SVPN } & 110 & 10 & 8 \\
& 50 & 21 & 20 \\
\multirow{2}{*}{ MD } & 113 & 1 & 0.7 \\
& 41 & 4.0 & 3.7 \\
\hline
\end{tabular}

Second, the equilibrium core structure of a dislocation at lowest energy state under Escaig stress is correctly calculated in the SVPN model by combining the steepest descent method and NEB method. At zero glide stress, the equilibrium dislocation core structure is obtained in two steps: (1) A stable state is obtained by the steepest descend method, and secondly the MEP for the dislocation overcoming Peierls barrier is computed by the NEB method, with the two ends in the NEB chain taking the stable state profile and its replica with a rigid shift of $\Delta x$, and the image with the lowest energy along the MEP is considered as the equilibrium state of the dislocation at the global energy minimum. (2) The equilibrium core 
structure at a non-zero Escaig stress can be successively obtained by the steepest descent method, by taking the equilibrium core structure at a slightly lower Escaig stress as the starting profile. Validity of this method for larger Escaig stress has been demonstrated in the MEP (Fig. 6b) for the two typical cases, where the dislocation state thus obtained actually stays in Peierls valley, the two ends at the upward-convex MEP path.

Last, the SVPN model can well capture details of interactions between the two partials and thereby the change of SFW (see Fig. 6a) as the full dislocation overcomes the Peierls barrier under Escaig stress, such as the different features of the interaction between the two partial dislocations at $\tau_{p}$ extremes. These features predicted by the SVPN model are all confirmed by MD simulations, as illustrated by similar variations of SFW in Fig. 6a, although the magnitude predicted by MD is slightly smaller than that by SVPN model.

These findings make the improved SVPN model one reliable method to predict dislocation core structure and Peierls stress under external loading, especially when empirical interatomic potential was unavailable for MD simulations, because inputs of the improved SVPN model can be acquired by combing first principles calculations and experiments. The anisotropic elastic constants needed to calculate the Stroh tensor for elastic energy, and the generalized stacking fault energy surface needed to calculate the misfit energy for a dislocation, can be obtained by first principles calculations (Jiang et al., 2016; Wang et al., 2016), and the gradient coefficient to calculate the gradient energy can be determined from the dislocation core structure, which in turn can be obtained by advanced experiments techniques (Wang et al., 2014b). 


\section{Conclusions}

Core structure and Peierls stress of dislocations in FCC metal under Escaig stress are comprehensively studied by the recently developed SVPN model (Liu et al., 2016). The results are validated by MD simulations, and are summarized as follows:

As Escaig stress increases, Peierls stress of the full screw and edge dislocation is quasi-periodically oscillating from a maximum value to a minimum, and the top-bottom difference is narrowing (see Fig. 3). These characteristics are well fitted by a formula consisting of a sinusoidal term times the exponentially decaying term plus a constant term. This formula provides a quantitative description of the effects of Escaig stress on the Peierls stress, building up a good basis for quantitative dependence of dislocation mobility on Escaig stress that will improve the mobility law for dislocations in dislocation dynamics simulations.

Local maximum (minimum) $\tau_{p}$ is reached when SFW is around integral (half-integral) multiples of Peierls period, where the two partials move in phase (out of phase) is responsible for the maximum (minimum) $\tau_{p}$. The repeated variation of SFW from integral to half-integral and half-integral to integral multiples of the Peierls period caused by the increasing Escaig stress is the reason for the periodically varying $\tau_{p}$, and weakening interaction between the two partials upon increasing SFW is the reason for the reducing gaps between the maximum and $\operatorname{minimum} \tau_{p}$

The improved SVPN model (Liu et al., 2016) has been demonstrated to be an efficient and powerful tool to study dislocation properties under external loading, e.g. the Escaig stress, as it is much more expedient than direct atomistic simulations and is capable of capturing the 
subtle details such as the interaction between the two partials as the full dislocation overcomes the Peierls barrier.

\section{Acknowledgments}

The authors acknowledge the support by the National Science Foundation of China Grant No. 51471107 and 51671132, CCS project Grant No. YK 2015-0202002, and the Center for High Performance Computing, Shanghai Jiao Tong University.

\section{References}

Amodeo, J., Dancette, S., Delannay, L., 2016. Atomistically-informed crystal plasticity in MgO polycrystals under pressure. Int. J. Plast. 82, 177-191.

Bacon, D., Barnett, D., Scattergood, R.O., 1980. Anisotropic continuum theory of lattice defects. Prog. Mater. Sci. 23, 51-262.

Baudouin, J.-B., Monnet, G., Perez, M., Domain, C., Nomoto, A., 2013. Effect of the applied stress and the friction stress on the dislocation dissociation in face centered cubic metals. Mater. Lett. 97, 93-96.

Bulatov, V., Cai, W., 2006. Computer simulations of dislocations. Oxford University Press Inc., New York, pp.75-77.

Bulatov, V., Kaxiras, E., 1997. Semidiscrete variational Peierls framework for dislocation core properties. Phys. Rev. Lett. 78, 4221.

Byun, T., 2003. On the stress dependence of partial dislocation separation and deformation microstructure in austenitic stainless steels. Acta Mater. 51, 3063-3071.

Byun, T.S., Hashimoto, N., Farrell, K., 2006. Deformation mode map of irradiated 316 stainless steel in true stress-dose space. J. Nucl. Mater. 351, 303-315.

Cai, W., Bulatov, V.V., Justo, J.F., Argon, A.S., Yip, S., 2000. Intrinsic mobility of a dissociated dislocation in silicon. Phys. Rev. Lett. 84, 3346.

Cheng, X., Shen, Y., Zhang, L., Liu, X., 2012. Surface effect on the screw dislocation mobility over the Peierls barrier. Philos. Mag. Lett. 92, 270-277.

Cui, Y., Po, G., Ghoniem, N., 2016. Temperature insensitivity of the flow stress in body-centered cubic micropillar crystals. Acta Mater. 108, 128-137.

Di Gioacchino, F., Quinta da Fonseca, J., 2015. An experimental study of the polycrystalline plasticity of austenitic stainless steel. Int. J. Plast. 74, 92-109.

Gröger, R., Vitek, V., 2013. Stress dependence of the Peierls barrier of $1 / 2<111>$ screw dislocations in bcc metals. Acta Mater. 61, 6362-6371.

Groh, S., Marin, E., Horstemeyer, M., Bammann, D., 2009. Dislocation motion in magnesium: a study by molecular statics and molecular dynamics. Model. and Simul. 
Mater. Sci. Eng. 17, 075009.

Healy, C.J., Ackland, G.J., 2014. Molecular dynamics simulations of compression-tension asymmetry in plasticity of Fe nanopillars. Acta Mater. 70, 105-112.

Henkelman, G., Jónsson, H., 2000. Improved tangent estimate in the nudged elastic band method for finding minimum energy paths and saddle points. J. Chem. Phys. 113, 9978.

Henkelman, G., Uberuaga, B.P., Jónsson, H., 2000. A climbing image nudged elastic band method for finding saddle points and minimum energy paths. J. Chem. Phys. 113, 9901.

Hirth, J., Lothe, J., 1982. Theory of Dislocations. John Wiley \& Sons Inc., New York, pp. 91, 461-463.

Jiang, Y., Wang, R., Wang, S., 2016. The temperature-dependent dislocation properties of aluminum from the improved Peierls-Nabarro model and first-principles. Philos. Mag. 96, 2829-2852.

Kamimura, Y., Edagawa, K., Takeuchi, S., 2013. Experimental evaluation of the Peierls stresses in a variety of crystals and their relation to the crystal structure. Acta Materia. 61, 294-309.

Kang, K., Yin, J., Cai, W., 2014. Stress dependence of cross slip energy barrier for face-centered cubic nickel. J. Mech. Phys. Solids 62, 181-193.

Lin, P., Liu, Z., Zhuang, Z., 2016. Numerical study of the size-dependent deformation morphology in micropillar compressions by a dislocation-based crystal plasticity model. Int. J. Plast. 87, 32-47.

Liu, G., Cheng, X., Wang, J., Chen, K., Shen, Y., 2016. Peierls stress in face-centered-cubic metals predicted from an improved semi-discrete variation Peierls-Nabarro model. Scripta Mater. 120, 94-97.

Lu, G., Bulatov, V.V., Kioussis, N., 2004. On stress assisted dislocation constriction and cross-slip. Int. J. Plast. 20, 447-458.

Lyu, H., Ruimi, A., Zbib, H.M., 2015. A dislocation-based model for deformation and size effect in multi-phase steels. Int. J. Plast. 72, 44-59.

Mishin, Y., Mehl, M., Papaconstantopoulos, D., Voter, A., Kress, J., 2001. Structural stability and lattice defects in copper: Ab initio, tight-binding, and embedded-atom calculations. Phys. Rev. B 63, 224106.

Nabarro, F., 1947. Dislocations in a simple cubic lattice. Proc. Phys. Soc. 59, 256.

Peierls, R., 1940. The size of a dislocation. Proc. Phys. Soc. 52, 34-37.

Rao, S.I., Dimiduk, D.M., El-Awady, J.A., Parthasarathy, T.A., Uchic, M.D., Woodward, C., 2015. Screw dislocation cross slip at cross-slip plane jogs and screw dipole annihilation in FCC Cu and Ni investigated via atomistic simulations. Acta Mater. 101, 10-15.

Salehinia, I., Bahr, D.F., 2014. Crystal orientation effect on dislocation nucleation and multiplication in FCC single crystal under uniaxial loading. Int. J. Plast. 52, 133-146.

Schoeck, G., 1994. The generalized Peierls-Nabarro model. Philos. Mag. A 69, 1085-1095.

Schoeck, G., 2012. The core structure and Peierls potential of dislocations in Al. Mater. Sci. 
Eng. A 558, 162-169.

Schoeck, G., 2013. Peierls stress and Peierls energy of a $70.5^{\circ}\langle 111\rangle$ dislocation in Mo. Philos. Mag. 93, 2363-2376.

Schoeck, G., Püschl, W., 1994. Dissociated dislocations in the Peierls potential. Mater. Sci. Eng. A 189, 61-67.

Shen, Y., Cheng, X., 2009. Dislocation movement over the Peierls barrier in the semi-discrete variational Peierls framework. Scripta Mater. 61, 457-460.

Shin, I., Carter, E.A., 2014. Simulations of dislocation mobility in magnesium from first principles. Int. J. Plast. 60, 58-70.

Srivastava, K., Gröger, R., Weygand, D., Gumbsch, P., 2013. Dislocation motion in tungsten: Atomistic input to discrete dislocation simulations. Int. J. Plast. 47, 126-142.

Stobbs, W., Sworn, C., 1971. The weak beam technique as applied to the determination of the stacking-fault energy of copper. Philos. Mag. 24, 1365-1381.

Stukowski, A., Cereceda, D., Swinburne, T.D., Marian, J., 2015. Thermally-activated non-Schmid glide of screw dislocations in W using atomistically-informed kinetic Monte Carlo simulations. Int. J. Plast. 65, 108-130.

Szelestey, P., Patriarca, M., Kaski, K., 2005. Dissociated dislocations in Ni: a computational study. Mater. Sci. Eng. A 390, 393-399.

Talonen, J., Hänninen, H., 2007. Formation of shear bands and strain-induced martensite during plastic deformation of metastable austenitic stainless steels. Acta Mater. 55, 6108-6118.

Taylor, R.L., 1972. On completeness of shape functions for finite element analysis. Int. J. Numer. Meth. Eng. 4, 17-22.

Ventelon, L., Willaime, F., Clouet, E., Rodney, D., 2013. Ab initio investigation of the Peierls potential of screw dislocations in bcc Fe and W. Acta Mater. 61, 3973-3985.

Vitek, V., 1968. Intrinsic stacking faults in body-centred cubic crystals. Philos. Mag. 18, 773-786.

Wang, J., Sehitoglu, H., Maier, H.J., 2014a. Dislocation slip stress prediction in shape memory alloys. Int. J. Plast. 54, 247-266.

Wang, S., Huang, L., Wang, R., 2016. The $90^{\circ}$ partial dislocation in semiconductor silicon: An investigation from the lattice $\mathrm{P}-\mathrm{N}$ theory and the first principle calculation. Acta Mater. 109, 187-201.

Wang, Z., Saito, M., McKenna, K.P., Ikuhara, Y., 2014b. Polymorphism of dislocation core structures at the atomic scale. Nature Communications 5, 3239.

Xiong, L., McDowell, D.L., Chen, Y., 2014. Sub-THz Phonon drag on dislocations by coarse-grained atomistic simulations. Int. J. Plast. 55, 268-278.

Xu, S., Guo, Y.F., Ngan, A.H.W., 2013. A molecular dynamics study on the orientation, size, and dislocation confinement effects on the plastic deformation of Al nanopillars. Int. J. Plast. 43, 116-127.

Yadav, S.K., Ramprasad, R., Misra, A., Liu, X.Y., 2014. Core structure and Peierls stress of edge and screw dislocations in TiN: A density functional theory study. Acta Mater. 74, 268-277. 
Yanilkin, A.V., Krasnikov, V.S., Kuksin, A.Y., Mayer, A.E., 2014. Dynamics and kinetics of dislocations in $\mathrm{Al}$ and $\mathrm{Al}-\mathrm{Cu}$ alloy under dynamic loading. Int. J. Plast. 55, 94-107.

Zhou, Q., Wang, J., Misra, A., Huang, P., Wang, F., Xu, K., 2016. Atomistic study of fundamental character and motion of dislocations in intermetallic Al2Cu. Int. J. Plast. 87, 100-113. 
Fig. 1. Illustration of a full (a) edge and (b) screw dislocation dissociating into two Shockley partials on (111) plane connected with stacking faults. The full dislocation has Burgers vector $1 / 2$ [-101], parallel to $z$ axis. Dislocations' line is either parallel to $x$ (for edge dislocation) or $z$ (for screw dislocation) axis.

Fig. 2. Variations of the slip profiles of the screw dislocation in $\mathrm{Cu}$ at zero Escaig stress with increasing glide stress $\tau_{y z}$. Peierls stress was determined as (a) 13 MPa by the improved SVPN model, and (b) 2. 9 MPa by MD simulations respectively.

Fig. 3. Variation of Peierls stress with increasing Escaig stress. (a) A screw and (b) an edge dislocation in $\mathrm{Cu}$. Predictions by the improved SVPN model and MD are fitted by Eq. (5) and shown in dashed curves.

Fig. 4. Dislocation density for the screw and edge dislocation at zero applied stress predicted by improved SVPN model and MD simulations.

Fig. 5. Deviation of SFW to its nearest (a) integral multiples of Peierls period for the $i_{t h}$ local maximum Peierls stress and (b) half-integral multiples of Peierls period for $i_{\text {th }}$ local minimum Peierls stress.

Fig. 6. Variations of (a) SFW and (b) dislocation energy for the screw dislocation moving one Peierls period $(\Delta x=\sqrt{3} b / 2)$ in copper for two representative cases when Peierls stress reaches local maximum and minimum respectively. Note that we plot SFW in (a) from 5.9 $\Delta \mathrm{x}$ to $7.9 \Delta \mathrm{x}$ to better illustrate the relatively small magnitude of variation of SFW. 


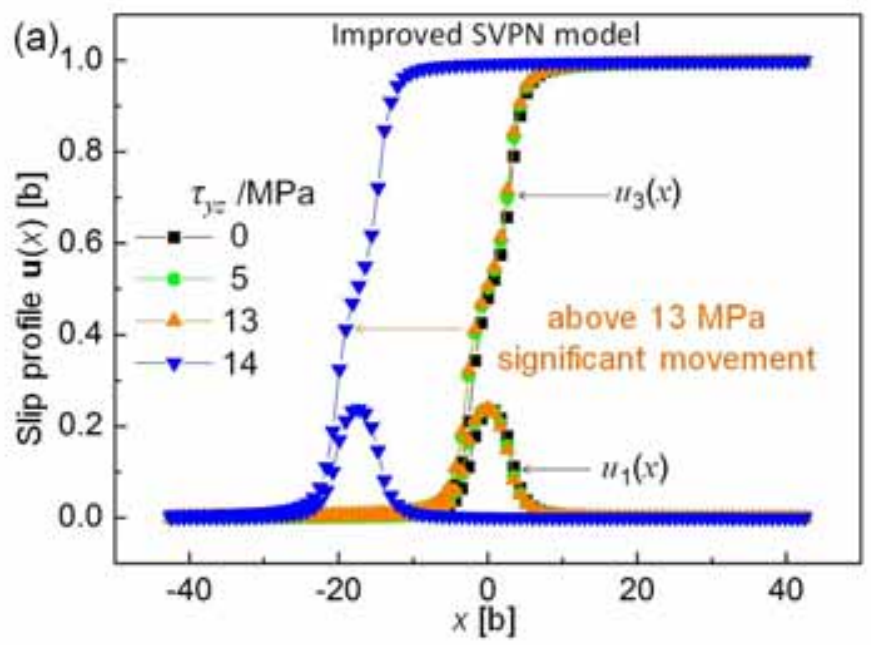




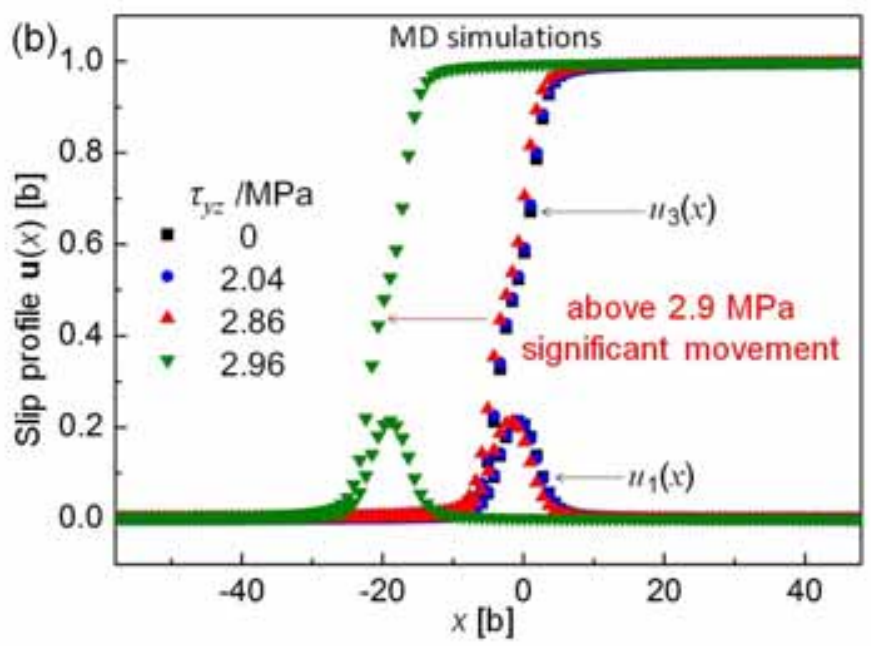




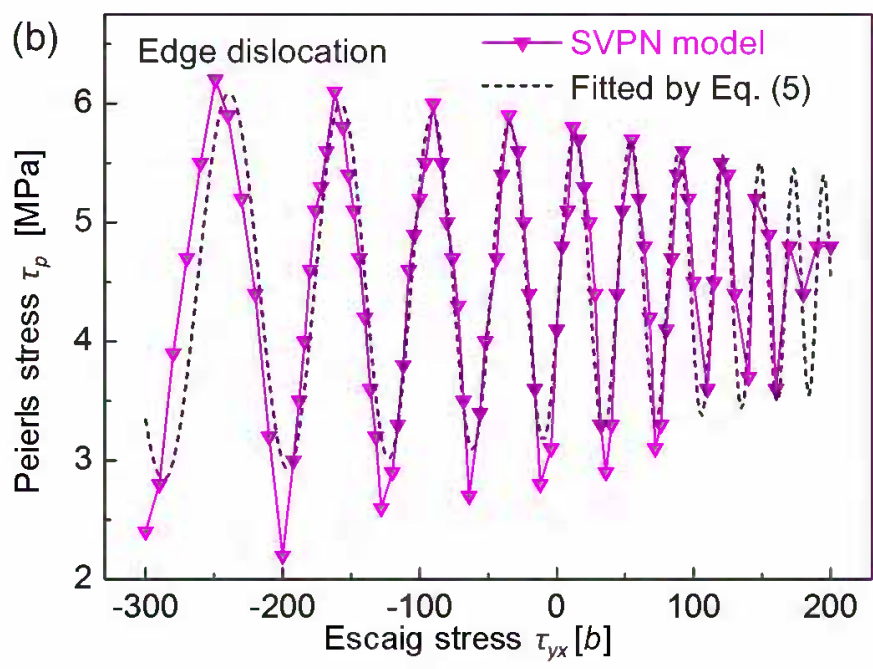




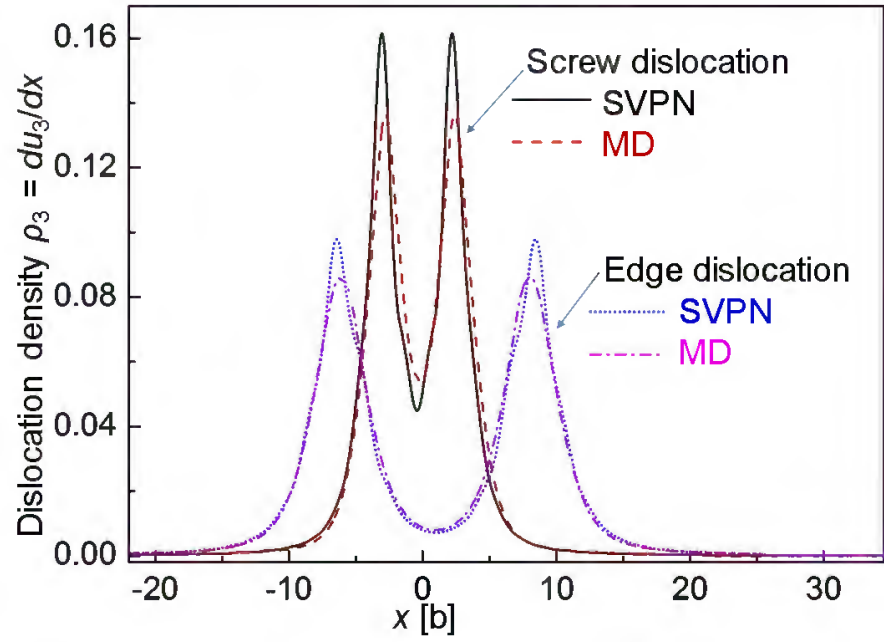



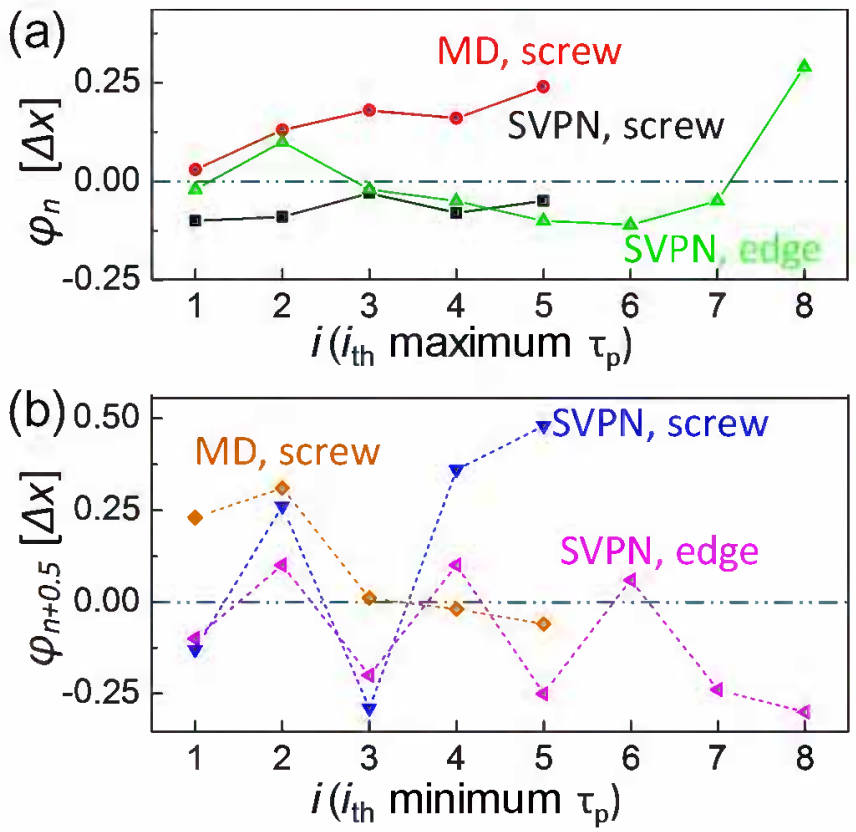


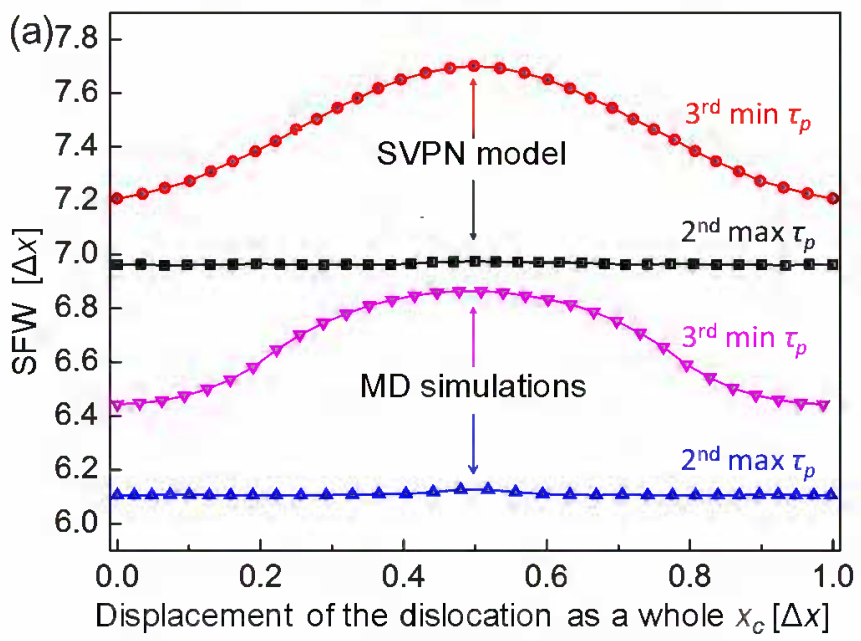




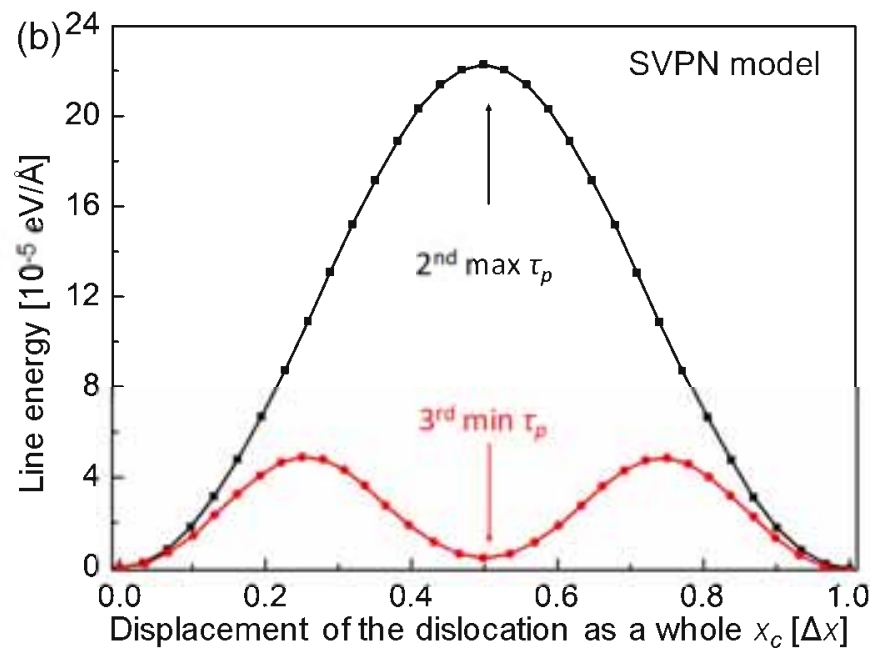


Table 1. Coefficients for the Escaig stress effects on Peierls stress in Eq. (5).

\begin{tabular}{ccccc}
\hline & $\tau_{0}[\mathrm{MPa}]$ & $\tau_{1}[\mathrm{MPa}]$ & $\alpha$ & $B$ \\
\hline edge, SVPN & 4.486 & 2.696 & 0.7278 & 81.42 \\
screw, SVPN & 16.42 & 11.55 & 0.7689 & 24.15 \\
screw, MD & 2.768 & -2.450 & 0.4451 & 21.68 \\
\hline
\end{tabular}

Table 2. Separation between the two partial dislocations at zero applied stress.

\begin{tabular}{cccc}
\hline & \multicolumn{3}{c}{$w$ or SFW (in units of b) } \\
\cline { 2 - 4 } & MD & Improved SVPN & Experiment value (Stobbs and Sworn, 1971) \\
\hline Cu, screw & 5.2 & 5.9 & $\sim 7.0$ \\
Cu, edge & 14.4 & 14.8 & $\sim 14.9$ \\
\hline
\end{tabular}

Table 3. Peierls stress for the screw dislocation in $\mathrm{Cu}$ under several Escaig stress $\tau_{y x}$.

\begin{tabular}{cccc}
\hline \multirow{2}{*}{$\tau_{y x}($ in MPa $)$} & \multicolumn{2}{c}{$\tau_{p}$ (in MPa) } \\
\cline { 3 - 4 } & & critical glide stress & NEB method \\
\hline \multirow{2}{*}{ SVPN } & 110 & 10 & 8 \\
& 50 & 21 & 20 \\
\multirow{2}{*}{ MD } & 113 & 1 & 0.7 \\
& 41 & 4.0 & 3.7 \\
\hline
\end{tabular}

